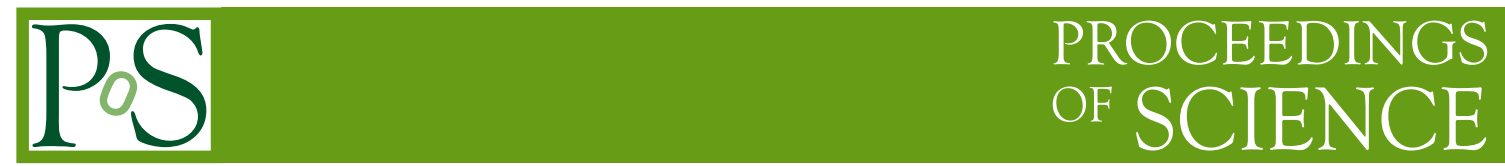

\title{
Darkside Status and Prospects
}

\author{
Charles Jeff Martoff*广 \\ Temple University \\ E-mail: cmartoffegmail.com
}

The DarkSide Dark Matter Search Program is a direct-detection search for dark matter using a Liquid Argon Time Projection Chamber. The detector is designed to be free of background, and background-rejection techniques have been developed to this aim. The current detector, DarkSide-50, has a 156 (36.9) $\mathrm{kg}$ total (fiducial) target of "underground argon", and is surrounded by a layered $20+1000$ ton active veto system. Projected developments for detectors of increasing size are briefly mentioned.

The European Physical Society Conference on High Energy Physics 5-12 July

Venice, Italy

* Speaker.

${ }^{\dagger}$ On behalf of the DarkSide Collaboration. 
The DarkSide Dark Matter Search Program

\section{What is DarkSide?}

DarkSide-50k is a direct-detection dark matter search experiment, based on a Liquid Argon Time Projection Chamber (LAr-TPC)[1, 2]. It is located at the Laboratorio Nazionale del Gran Sasso (LNGS) in Italy. Its goal is to perform a background-free dark matter search using lowradioactivity argon extracted from underground sources (UAr), and to act as a prototype for planned much larger LAr detectors in the DarkSide program [3].

\subsection{The detection mechanism}

A nuclear recoil produced by the scattering of a WIMP from an Ar nucleus (Nuclear Recoil, NR) ionizes the liquid argon and produces scintillation light. The resulting prompt light signal is called S1. The ionized electrons are drifted through the liquid argon towards a gaseous argon region, where they induce electroluminescence, resulting in a second, delayed light signal (S2).

Knowing the drift velocity of electrons in LAr, the time difference between S1 and S2 gives the vertical position of the WIMP-nucleus interaction. The spatial distribution of S2 light allows the $\mathrm{x}-\mathrm{y}$ position of each event to be obtained, resulting in a 3D reconstruction of the event positions.

\subsection{The LAr-TPC}

\subsubsection{Description of the TPC}

The core of the experiment, the LAr-TPC, is a cylindrical volume containing 153 (36.9) kg of total (fiducial) UAr. Scintillation light is detected in 38 Hamamatsu 3" low-background, highefficiency PMTs, 19 on the top of the cylinder and the rest on the bottom. The PMTs are submerged in the liquid argon, viewing the LAr active volume through fused silica windows, which are coated with conductive indium tin oxide (ITO). The ITO allows the inner window faces to act as grounded anode (top) and -HV cathode (bottom) electrodes, which along with a field cage, define the TPC drift field.

The walls of the active volume are $25 \mathrm{~mm}$ thick PTFE coated with vacuum-evaporated TPB wavelength shifter. The S1 and S2 light is emitted at $128 \mathrm{~nm}$, so the TPB is needed to shift it to $420 \mathrm{~nm}$, the peak sensitivity wavelength of the PMTs. The photoelectron (PE) yield has been determined to be $7.0 \pm 0.3 \mathrm{PE} / \mathrm{keVee}$ at $200 \mathrm{~V} / \mathrm{cm}$. Exceptionally low noise is achieved by the use of cryogenic amplifiers mounted on the PMT's and immersed in the LAr.

\subsubsection{Pulse Shape Discrimination}

The bulk of the background for the DarkSide-50 experiment is from ordinary radioactivity, producing ionizing electron recoils (ER). This can be identified and rejected by looking at the shape (vs. time) of the S1 signal of each event. Electron-recoil background events (typically $\beta / \gamma$ radiation) have S1 shape very different from that of NR (see Figure 1). The pulse shape discrimination (PSD) parameter $f_{90}$ - defined as the fraction of $\mathrm{S} 1$ signal detected in the first $90 \mathrm{~ns}$ from the trigger - has been shown to discriminate between NR events and ER events at the level of $2 \cdot 10^{-7}[1]$. 

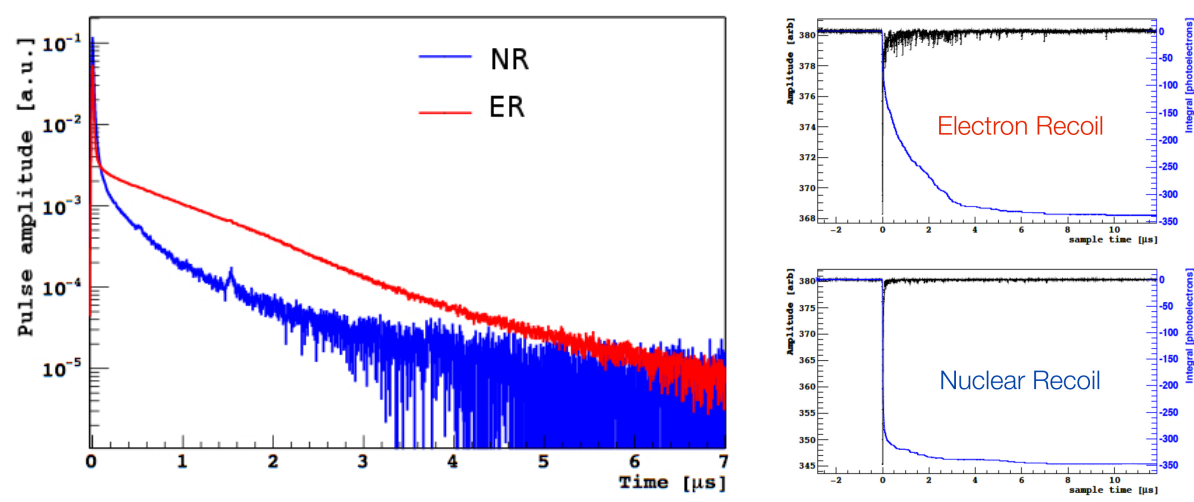

Figure 1: (Left) Amplitude of an S1 signal for an ER and an NR. (Right) Waveforms of an electron and a nuclear recoil, superimposed with integrated charge.

\subsubsection{Underground Argon}

PSD and other background-rejection techniques were perfected using Atmospheric Argon (AAr), which contains $\sim 1 \mathrm{~Bq} / \mathrm{kg}$ of the cosmic-ray-produced ${ }^{39} \mathrm{Ar}$ isotope [1]. The argon currently used in the active volume is low-radioactivity Underground Argon (UAr). It was extracted from a natural gas well in Colorado, purified of $\mathrm{He}$ and $\mathrm{N}_{2}$ impurities at Fermilab, and finally shipped to LNGS. UAr has a measured ${ }^{39}$ Ar activity of $0.73 \pm 0.11 \mathrm{mBq} / \mathrm{kg}$, about 1400 times less than AAr.

Small contaminations from ${ }^{85} \mathrm{Kr}(0.73 \pm 0.11 \mathrm{mBq} / \mathrm{kg})$, and ${ }^{37} \mathrm{Ar}\left(2.4 \mathrm{keV}\right.$ Auger, $\left.t_{1 / 2}=35 \mathrm{~d}\right)$ were also discovered, but their contributions either decay rapidly, or can be eliminated by cryogenic distillation in future DarkSide detectors (Depleted Argon, or DAr, see below).

\subsubsection{Calibration}

An in situ calibration campaign has been performed in DarkSide-50 using radioisotope neutron sources. A particle identification histogram (S1 pulse height vs. $f_{9} 0$ ) for TPC events coincident with AmBe neutron source events in the LSV is plotted in Figure 2. The high rate, accompanying gamma rays and inelastic scattering of energetic neutrons from this AmBe neutron source are responsible for the events scattered between the neutron and electron recoil bands.

\subsection{Veto system}

\subsubsection{Description of the veto}

To reject residual neutron and cosmic ray background at Gran Sasso depth, the TPC is enclosed in a $4 \mathrm{~m}$ diameter stainless steel sphere, holding 30 tonnes of borated liquid scintillator [5], which constitutes the Liquid Scintillator Muon Veto (LSV). The scintillator is composed of pseudocumene (PC) with 5\% trimethyl borate (TMB), with Diphenyloxazole (PPO) wavelength shifter.

110 8" low-radioactivity, high-quantum-efficiency Hamamatsu PMTs mounted on the inner surface of the sphere detect the scintillation light. Signals are produced mainly by the ${ }^{10} \mathrm{~B}(n, \alpha){ }^{7} \mathrm{Li}$ neutron-capture reaction, which usually produces a $478 \mathrm{keV}$ gamma-ray, as well as by elastic scattering and capture interactions with the hydrocarbon scintillator. The measured PE yield of the 


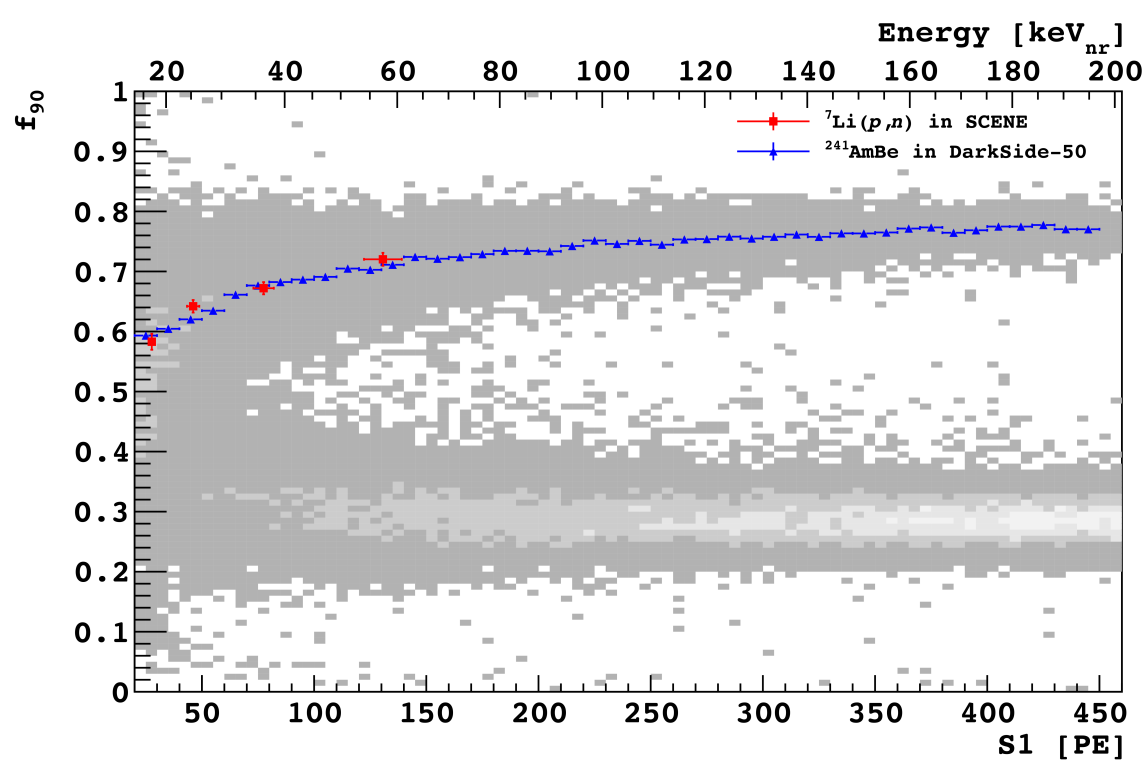

Figure 2: Data from the in situ AmBe calibration. The red points show the mean NR signal characteristics determined in the ScENE calibration experiment [4].

LSV is $0.6 \pm 0.1 \mathrm{PE} / \mathrm{keV}$. The neutron veto efficiency of the LSV was determined from AmBe and $\mathrm{Am}^{1} 3 \mathrm{C}$ neutron source runs to be $99.85 \pm 0.26 \%$ for neutrons interacting in the TPC.

The LSV itself is lies within an $11 \mathrm{~m}$ diameter, $10 \mathrm{~m}$ high cylindrical tank filled with high purity water for detection of Cherenkov radiation from cosmic ray muons (Water Cherenkov Detector, or WCD). In Ref.[2], the event was classified as a cosmogenic neutron based on its WCD and LSV signals.

\section{Comparison to Liquid Xenon}

One might ask why continue to pursue LAr dark matter searches when the LXe experiments [6] have already reached the multi-tonne phase. In fact, LAr has advantages compared to LXe when unaviodable backgrounds from solar and Diffuse Supernova Background (DSNB) neutrinos are considered.

The ER rate from solar neutrinos is the same in dru for LAr and LXe $\sim 40000$ events/1000 ton $\cdot \mathrm{yr}=9 \cdot 10^{-6} \mathrm{dru}$. However, the leakage of solar neutrino events past the NR discrimination in LXe is of order $2 \cdot 10^{-3}$ [7], which gives about 100 solar $v$ events in the WIMP ROI per 1000 ton. yr, while the leakage in an advanced LAr detector will be closer to $<6 \cdot 10^{-8} \simeq 0.05$ solar $v$ events in the WIMP ROI per 1000 ton- yr. The limited discrimination in LXe may allow other sources of ER background to limit the sensitivity at even higher levels.

Coherent scattering of solar and DSNB neutrinos produces a nuclear recoil background which cannot be distinguished from a WIMP signal in conventional (non-direction-sensitive) detectors. This has been referred to as the "neutrino floor", the ultimate limit to WIMP sensitivity with current technology. Although the coherent WIMP-nucleon scattering event rate per ton- yr of LAr exposure is only $\sim 0.25$ of that for LXe (it has a dependency $\frac{(A-Z)^{2}}{A}$ ), the neutrino floor event rate is also lower in LAr by the same factor. 


\section{DarkSide-20k}

The successor of DarkSide-50 will be DarkSide-20k [3]. Proposed to INFN and NSF in 2015, it will feature:

- 20 tonne LAr fiducial mass;

- new LSV and WCD veto system;

- new UAr and DAr plants to use active isotope depletion by cryogenic distillation, producing the requisite amount of DAr with even lower ${ }^{39}$ Ar levels than the UAr feedstock.

This experiment is designed to achieve a background-free exposure of 100 ton- yr, and to reach a sensitivity of $\sigma(W N)<10^{-47} \mathrm{~cm}^{2}$ for a WIMP mass of $1 \mathrm{TeV} / \mathrm{c}^{2}$. Figure 3 shows the projected sensitivity limits for DS-20k compared to other experiments.

Still in the initial planning stages is ARGO, an even larger device designed to reach the neutrino floor with an exposure of 1000 ton-yr.

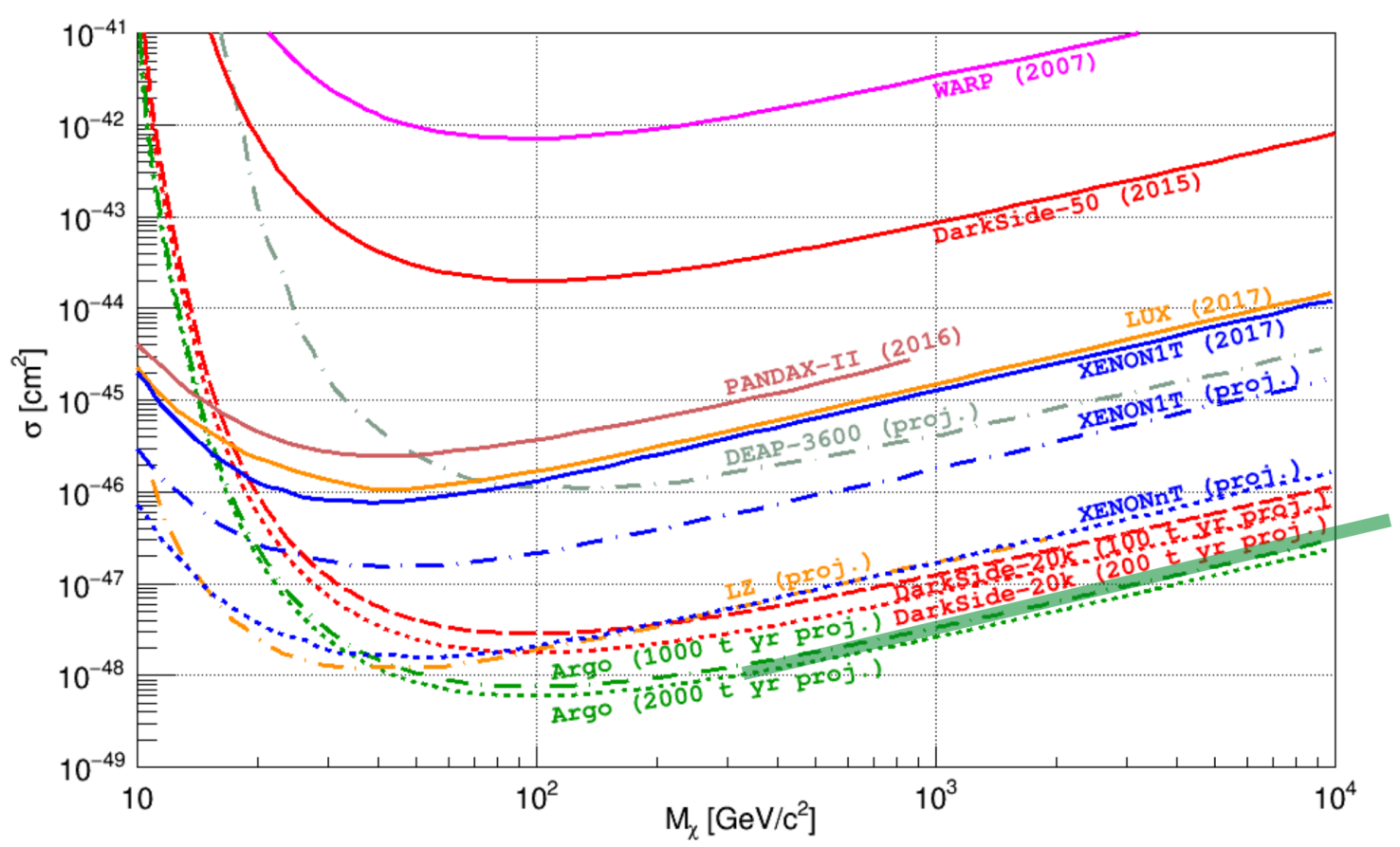

Figure 3: Present and projected experiments' sensitivities.

\section{References}

[1] P. Agnes et al. First results from the DarkSide-50 dark matter experiment at Laboratori Nazionali del Gran Sasso. Physics Letters B, 743:456 - 466, 2015.

[2] P. Agnes et al. Results from the first use of low radioactivity argon in a dark matter search. Phys. Rev., D93(8):081101, 2016. [Addendum: Phys. Rev.D95,no.6,069901(2017)]. 
[3] C. E. Aalseth et al. DarkSide-20k: A 20 Tonne Two-Phase LAr TPC for Direct Dark Matter Detection at LNGS. 2017.

[4] H. Cao et al. Measurement of Scintillation and Ionization Yield and Scintillation Pulse Shape from Nuclear Recoils in Liquid Argon. Phys. Rev., D91:092007, 2015.

[5] P. Agnes and others. The veto system of the DarkSide-50 experiment. Journal of Instrumentation, 11(03):P03016, 2016.

[6] D. S. Akerib et al. Results from a Search for Dark Matter in the Complete LUX Exposure. Phys. Rev. Lett., 118:021303, Jan 2017.

[7] E. Aprile et al. Physics reach of the XENON1T dark matter experiment. Journal of Cosmology and Astroparticle Physics, 2016(04):027, 2016. 